\title{
A Swing to Intermittent Clean Self- catheterisation as a Preferred Mode of Management of the Neuropathic Bladder for the Dextrous Spinal Cord Patient
}

\author{
V. B. Hill, MBBS, BPhty, FACRM, W. E. Davies AM, FRACP, \\ FACRM \\ Spinal Injuries Unit, Princess Alexandra Hospital, Brisbane, Queensland, \\ Australia.
}

\section{Summary}

Since 1977, 301 patients with spinal cord pathology have been taught to self-catheterise. The majority (234) remain on the programme. The reasons for the 67 ceasing the procedure and the changing pattern of bladder management amongst Queensland's spinally injured community are discussed. The complications and advantages of the system are considered and a plea is put forward that intermittent clean selfcatheterisation should be proferred as the preferred option to all spinally injured persons with good hand function.

Key words: Self-catheterisation; Spinal cord injury; Long term follow-up

Intermittent clean self-catheterisation (ICSC) has gradually gained acceptance since Lapides presented his first paper in 1972 (Lapides et al.). Those managing the meningomyelocoele (Light and Van Blerk, 1977; McIlroy, 1977) patients adopted the technique when the long term shortcomings of ileal diversion became apparent. In the late 1970s, those attempting to 'train' paraplegic females (Madersbacher and Diokno, 1977) recognised that many were not prepared to discipline themselves sufficiently for the technique to be successful; these patients were putting up with being wet, or accepting an indwelling catheter for convenience. ICSC used in conjunction with anticholinergic therapy proved successful in these patients, who have shown low infection rates, have rarely developed calculi, and frequently have had significant improvement in their upper urinary tracts. Few patients developed strictures, and the procedure has found a high acceptance level amongst patients.

Despite these obvious advantages, many Spinal Injuries Units still persist with conventional bladder training and the use of penile sheaths and leg bags. They not infrequently need to resort to transurethral external sphincterotomy to achieve satisfactory bladder emptying.

Correspondence to: Dr V. Hill, Spinal Injuries Unit, Princess Alexandra Hospital, Ipswich Road, Wolloongabba, Brisbane, Queensland, Australia. 
The literature includes many accounts of successful ICSC trials (Lapides et al., 1972; Light and Van Blerk, 1977; MacGregor and Diokno, 1979; Maquire and Savastano, 1983; Maynard and Diokno, 1982). This paper documents the pattern of change in the management of neurogenic bladders in the Queensland Spinal Injuries Unit over the past 10 years.

\section{Methods and materials}

The urological status of all patients is assessed during acute admission using urodynamics, intravenous pyelogram, and micturating cystogram. Where indicated, cystoscopy was performed. They are all followed up annually with an intravenous pyelogram for the first 3 years; if no abnormality is detected, they are then followed annually with an ultrasonic scan of the kidneys and bladder. Whilst on the unit, urine microscopy and culture is performed weekly. After discharge, patients are encouraged to seek regular review of their urine through their general practitioner. After the first year, check urine microscopy and culture is generally done only if the patient is having problems, or when he attends Outpatients for annual review. All paraplegic patients are commenced on anticholinergic medication if urodynamics indicate a hyperreflexic detrusor response. Tetraplegic patients with lesions at the level of the 6th cervical segment and below, can be taught to perform ICSC, but since 1986, those with less than Grade 2 triceps have been discouraged from trying, as the failure rate of this group in the long term has been high.

While in the ward, patients catheterise themselves, using a sterile No. 14 Nelaton catheter. After discharge they are taught to reuse catheters which are soaked in dilute aqueous Hibitane after use. The catheter is passed from a plastic bag to which a 'blob' of lubricant is added. Twelve catheters are issued to each patient every 3 months.

Patients are taught four basic principles:

1. To drink sufficient fluid to ensure that they have between 300 and $500 \mathrm{ml}$ of urine in their bladders every 4 hours.

2. To catheterise fourth hourly (a 6 hour break at night is allowed).

3. To empty their bladders completely each time the catheter is passed.

4. To ensure that they are careful with their catheter maintenance and the technique of insertion.

Of the 301 patients who commenced ICSC training, the neurological deficit was of traumatic origin in 273 and due to medical causes in 28 . Of the 42 females, 20 had complete cord lesions and 22 were incomplete. The 259 males comprise 115 complete and 144 incomplete spinal lesions. Forty four of those taught ICSC were tetraplegics and 277 paraplegics. The eldest was 81 years of age and the youngest 11 . The mean age was 23 and $52^{\circ}{ }_{0}$ were under the age of 25.

\section{Results}

Since 1977, 301 patients with spinal cord pathology have been taught ICSC, with 234 of these remaining on the programme. Of the 67 who have ceased to catheterise, 18 now void normally, 13 patients have died (the majority over the 


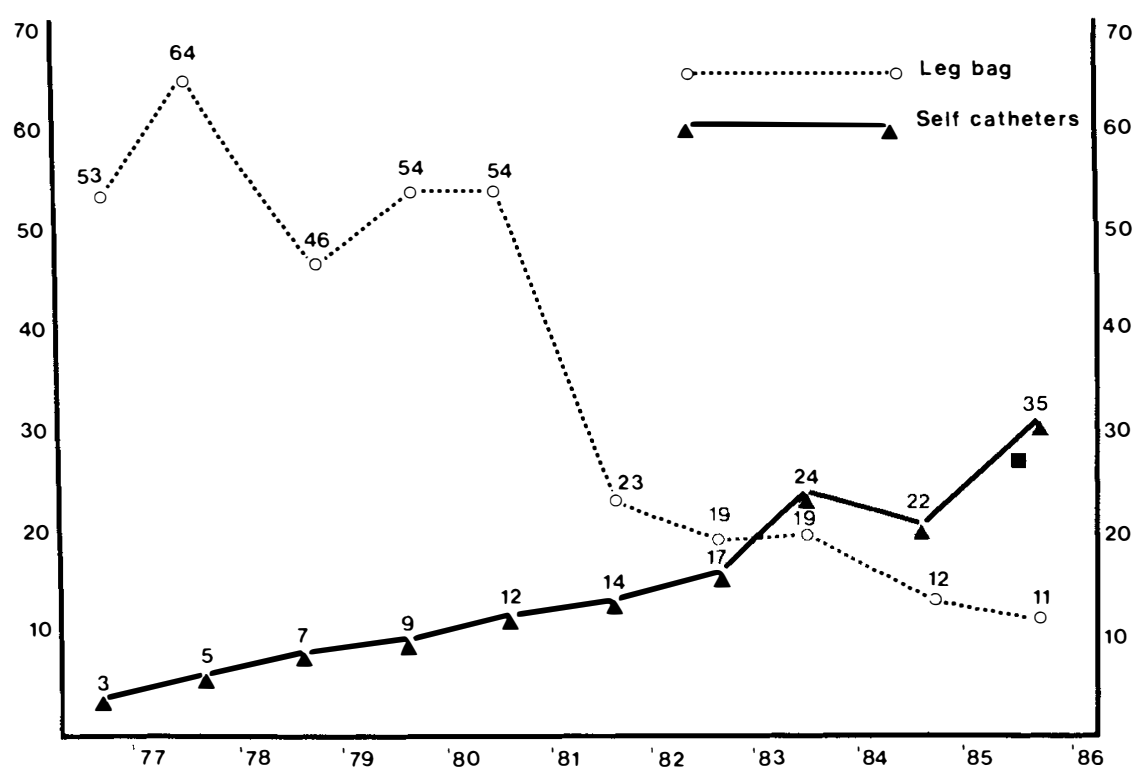

Figure 1 Numbers of patients discharged on self-catheters or with a leg bag 1977-1986

age of 60), 22 patients reverted to the use of leg bags, and of the latter, 17 are C6 tetraplegics who persevered for several weeks, but found the technique too time consuming. Five patients were unable to remain dry between catheterisation and elected to use external collecting devices. Two of these patients required an external urethral sphincterotomy. Ten patients reverted to indwelling catheters. Five were over the age of 60,2 became pregnant, and 1 after a cerebrovascular accident was unable to continue ICSC at his nursing home. One Aborigine preferred an indwelling catheter. Four patients have been lost to follow-up.

\section{Medication}

Of the 234 patients still on the programme, 42 currently require no drug therapy; all have hyporeflexic bladders as demonstrated by urodynamics, 15 having lower motor neurone lesions. To prevent spontaneous micturition, 125 take penthienate bromide (10-40 mg per day), 40 take amitriptyline (30-75 mg/day) and 24 take a combination of both these. Two patients require pseudoephedrine in addition. Four patients use phenoxybenzamine at times to facilitate catheterisation when spasm of the external urethral sphincter causes difficulty.

Figure 1 demonstrates the changes in the number of patients discharged on ICSC between 1977 ( 3 people) and 1986 (35 people). In all, 148 patients were taught the technique during their acute admission in the 10-year period. During this same period, 355 patients were discharged with leg bags, the number decreasing from 53 in 1977 to 11 in 1986.

Figure 2 shows the breakdown into paraplegics and tetraplegics of those patients who were discharged with leg bags.

Figure 3 illustrates the number of external urethral sphincterotomies per- 


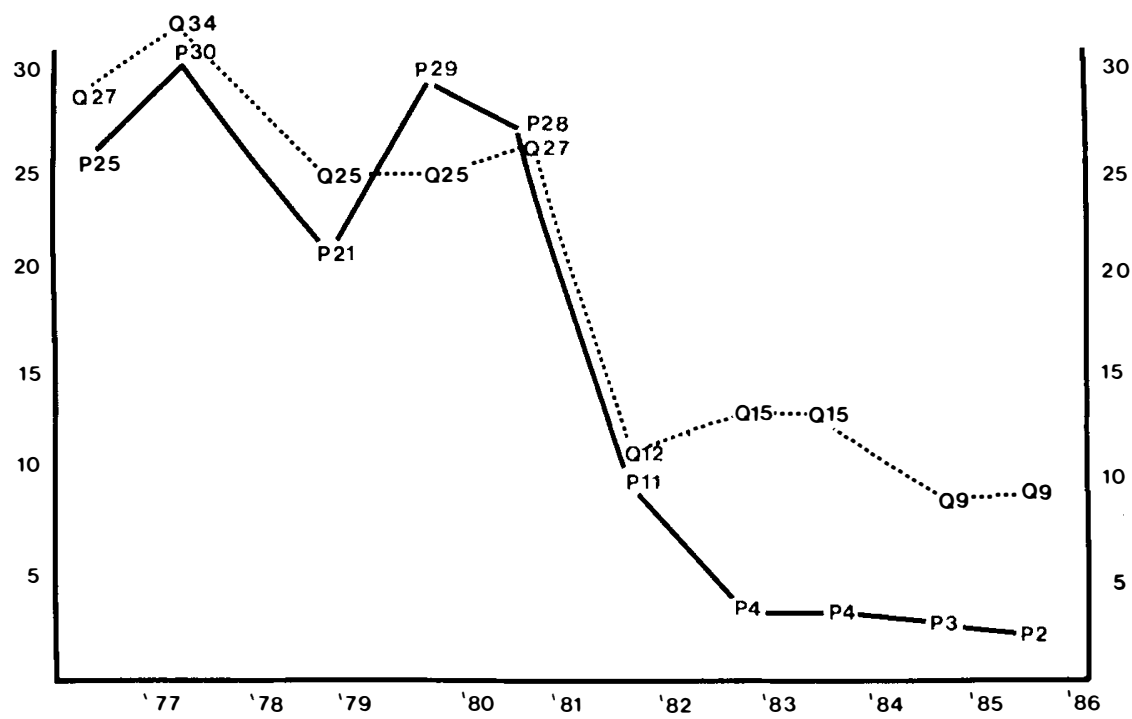

Figure 2 Breakdown of the leg bag group into paraplegics and tetraplegics 1977-1986

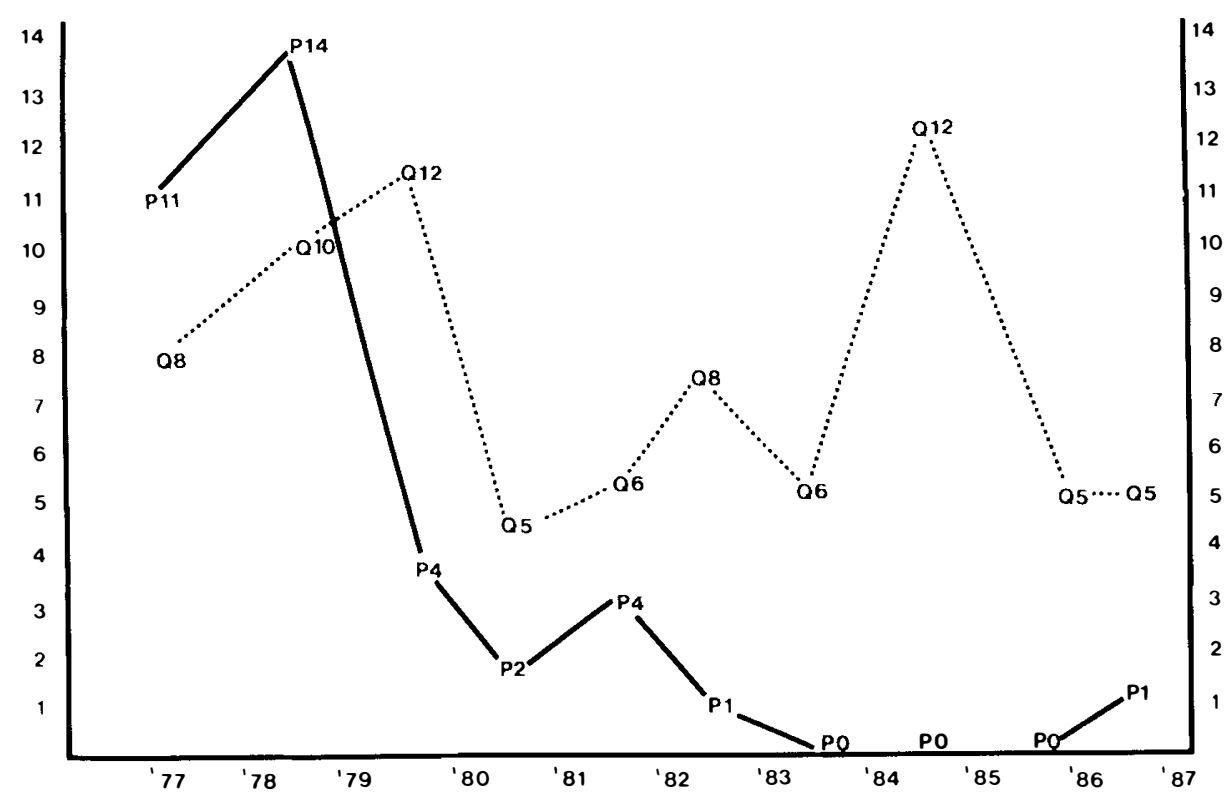

Figure 3 External urethral sphincterotomies 1977-1986 paraplegics and tetraplegics

formed between 1977 and 1986, and demonstrates the almost complete elimination of this operation in paraplegic patients over the last 6 years. The incidence among tetraplegics has dropped, but not so dramatically. During this 10 -year period, the number of acute admissions has increased by approximately $5 \%$ annually. 


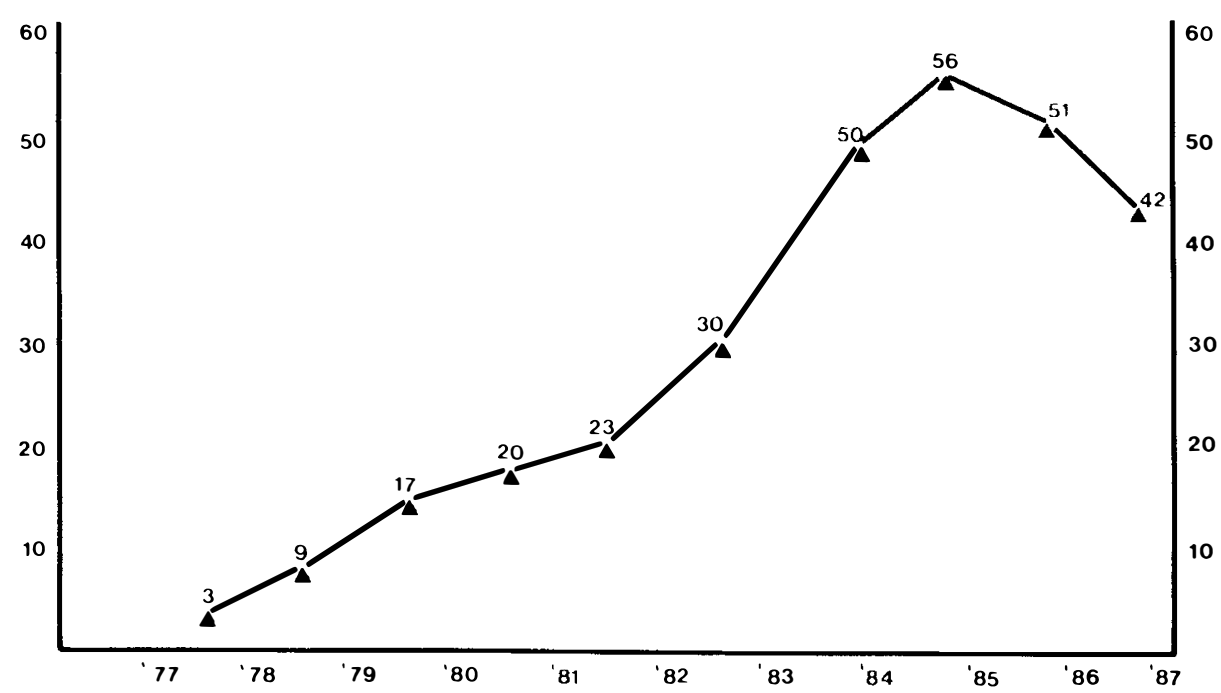

Figure 4 Total number of patients taught self-catheterisation 1977-1986 = 301 patients

Figure 4 records the total number of patients taught to self-catheterise from 1977-1986. The peak in the graph between 1983 and 1985 reflects the 86 patients who had originally been discharged using leg bags and were readmitted to be taught ICSC. Sixty eight of these patients requested the change, while 18 accepted the offer of ICSC rather than undergo external urethral sphincterotomy, because of evidence of deterioration in their upper tracts on follow up X-rays.

\section{Complications}

Two patients required admission to the ward for treatment of systemic urinary tract infection. One patient developed a renal calculus and 3 developed vesical calculi. Of 15 patients who presented with orchitis, 10 required admission to hospital. Two suffered recurrent orchitis, and a vasectomy was performed on the infected side of each. One patient required orchidectomy. Bacteriuria with pyuria of less than 10 per HPF was not treated with antibiotics, unless there was evidence of systemic infection.

At the time of commencing ICSC and anticholinergic therapy, routine cystography demonstrated urinary tract reflux in 20 patients. Subsequent micturating cystourethrograms have shown that reflux was no longer present on strain views. Figures $5 \mathrm{~A}$ and $5 \mathrm{~B}$ illustrate this situation in 1 patient.

\section{Discussion}

Operant conditioning plays an important role in the success of the ICSC programme. Just as patients learn to be fastidious with their bowel regimen in order to avoid bowel accidents, so too those who self-catheterise find that they have to be meticulous with their bladder management if they are to remain continent between catheterisations. ICSC teaches the patient a great deal about 

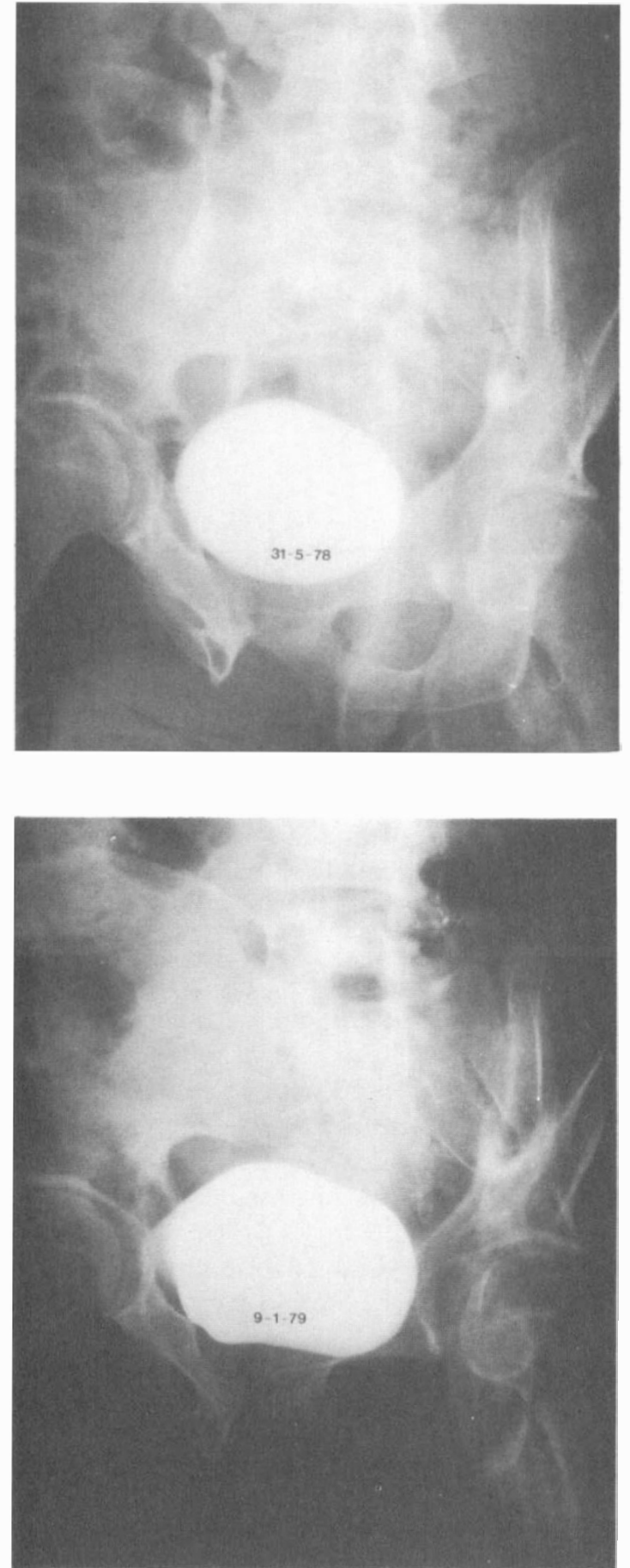

Figure 5A and 5B Cystograms before and some months after commencing ICSC in 1 patient. 
the physiology of his kidneys and bladder, whereas those patients who wear a urinary collecting device tend to let their urine 'run'. The moist environment within a condom is conducive to the growth of gram negative organisms, whereas the dry glans penis of the patient on self catheterisation perhaps deters infective agents.

\section{Advantages to the patient}

The most obvious advantage is the lack of the external collecting device. Activities such as swimming and sexual encounters are greatly enhanced. A patient's potential fertility is greater if he has not undergone an external sphincterotomy. Female patients who are bladder trained, generally cannot empty their bladders satisfactorily by tapping, unless they have a reasonable volume of urine in their bladders. The ability to empty the bladder completely when the opportunity arises is considered an advantage by many of them.

\section{Advantages to the health care system}

If each catheter is reused for a period of 1 week, the cost of maintaining a patient on ICSC is one fifth of the sum needed to maintain a patient with urinary drainage apparatus. No more than 3 days are required to train a patient to self-catheterise, whereas bladder training can take weeks and even months. This time differential has therefore led to significant reduction in the time acute patients spend in hospital.

It is rare for patients on ICSC to come back with urological complications, whereas $20-30 \%$ of patients who have undergone external sphincterotomy require a further revision of the procedure at least once over a 10-year follow-up period (Vaughton and Low, 1986).

\section{Problems encountered}

The most common problem has been incontinence between catheters. This is generally the result of infection or failure to catheterise often enough to avoid overdistension. Patients are instructed to increase their anticholinergic therapy, and their fluid intake, and at the same time to catheterise more frequently. If the problem persists, it has been necessary to insert a catheter for 3-4 days and increase the anticholinergic therapy to the point where the patient becomes thirsty, but does not complain of blurred vision. Once the result of the urine culture is known, the catheter is removed and the patient commenced on an appropriate antibiotic.

Difficulty with the insertion of the catheter has occurred from time to time. The patient is taught to leave the catheter at the level of the sphincter for a minute, and then try to ease it in gently. If this fails he is advised to insert a gloved finger into the anus, which will usually relieve the problem. If the difficulty recurs, a slightly stiffer catheter is used, and on a few occasions, a Coudé tipped catheter has been found necessary. Before resorting to this, the patient is given phenoxybenzamine, which is taken as soon as he first notices a tightening of the sphincter. It is seldom necessary to continue this therapy for more than $2-3$ days. 
A major difficulty still exists for those who have an inadequate bladder capacity (less than $350 \mathrm{ml}$ ). The present management of this group has been to revert to condom drainage. One patient has undergone successful bladder augmentation (Mitchell and Baches, 1982). It is anticipated that this solution will be offered in the future to many of those patients currently taking high doses of anticholinergic drugs.

\section{Conclusion}

This paper proposes that an external urethral sphincterotomy should be 'a last resort' when managing patients with spinal cord damage and good hand function. Sexual function and fertility are aspects of management which are now receiving much more attention in all spinal units, and self-catheterisation certainly improves self image and sexuality, and may even improve the quality of semen produced. Intermittent clean self-catheterisation is the preferred option for all patients with adequate hand function.

\section{Acknowledgements}

The authors wish to thank the following for their encouragement and assistance: Dr Graham Holmes, MBBS, FRCSE, FRACS, Dr Ross Cartmill, MBBS (Qld) FRCS, FRACS, and Dr Peter Heathcote, MBBS (Qld), FRACS (Urol).

\section{References}

Boles BM, Mara wu MN, Porteus T 1978-1979 Two technical aids for the intermittent sterile catheterisation. Paraplegia 16:303-305.

Diokno AC, SoNDA LP, Hollander JB, et al. 1983 Fate of patients started on clean intermittent self catheterisation therapy ten years ago. American fournal of Urology 129:1120-1122.

LAPIDES J, Diokno AC, Silbert SJ, et al. 1972 Clean intermittent self catheterisation in the treatment of urinary tract disease. Fournal of Urology 107:458.

LIGHT, K, VAN BLERK PJP, 1977 Intermittent catheterisation in congenital neurogenic bladder: a preliminary report. British fournal of Urology 49:523-526.

MACGREGOR RJ, Diokno AC 1979 Self catheterisation for decompensated bladder, a review of 100 cases. Fournal of Urology 122:602-603.

MADERSBACHER H, WEISSTEINER G 1977 Intermittent self catheterisation. An alternative to the treatment of neurogenic urinary incontinence in women. European Urology 3:82-84.

MAQUIRE EJ, SAVASTANO JA 1983 Long term follow up of spinal cord injury patients managed by intermittent catheterisation. American fournal of Urology 129:775-776.

MAYNARD MF, DIOKNO AC 1982 Clean intermittent catheterisation for spinal cord injury patients. Fournal of Urology 128:477-480.

MCILROY RF 1977 Intermittent non sterile catheterisation of children suffering from spina bifida. An alternative to urinary diversion. Australian and New Zealand fournal of Surgery 47(6):754757.

Mitchell ME, BACHes DJ 1982 Intermittent clean self catheterisation in children with bladder augmentation. Annual meeting of American Urological Association, Kansas City (Abstract 77).

VAUGHTON KC, Low AI 1986 Urodynamic assessment of the use and effectiveness of sphincterotomy in paraplegics. Australian Urological Society meeting, Perth. 